\title{
Micromanaging regeneration
}

\author{
Elly M. Tanaka ${ }^{1,2}$ and Gilbert Weidinger ${ }^{1,3,4}$ \\ ${ }^{1}$ Center for Regenerative Therapies, 01307 Dresden, Germany; ${ }^{2}$ Max Planck Institute for Molecular Cell Biology and \\ Genetics, 01307 Dresden, Germany; ${ }^{3}$ Biotechnology Center of the Technical University Dresden, 01307 Dresden, Germany
}

All adult animals are capable of maintaining their shape and function by homeostatic replacement of dying cells, but their ability to regenerate lost or damaged organs and appendages varies widely. Unfortunately for us humans, mammals are found at the lower end of the vertebrate spectrum. We can regrow large parts of the liver and pancreas, repair limited damage to skeletal muscle and peripheral nervous system, but pale in comparison with the amazing capacity of amphibia and fish to repair most organs, including lens, retina, heart muscle, and CNS, and to even regrow amputated limbs and fins. After more than 100 years of research on regenerative phenomena, it is still a mystery why these lower vertebrates can do what we can't. Nevertheless, recent research using molecular tools has been increasingly successful in uncovering the molecular mechanisms that control regeneration. A study by Yin et al. (2008) in this issue of Genes \& Development now adds microRNAs to the picture. They show that expression of many microRNAs changes during zebrafish tail fin regeneration and that FGF signaling promotes proliferation of progenitor cells in the fin by suppressing expression of microRNA-133, which inhibits translation of $m p s 1$, a kinase required for cell proliferation.

\section{Regenerating adult tissues retain cell plasticity}

Zebrafish have become a very successful model for both regeneration and microRNA biology. Amputation of the tail fin triggers a particularly robust and efficient regenerative process. Within $2 \mathrm{wk}$, bone, connective tissue, blood vessels, nerves, epidermis, and pigmentation are restored and the fin has grown back to its exact preamputation length (Poss et al. 2003; Stoick-Cooper et al. 2007a). Fin regeneration shares many characteristics with limb regeneration in urodele amphibians (salamanders and newts), including the formation of a proliferative mass of progenitor cells-the so-called blastema-that gives rise to all differentiated cell types during regeneration. In amphibia, it appears that the blastema forms by both the intriguing capability of differentiated

\footnotetext{
${ }^{4}$ Corresponding author.

E-MAIL gilbert.weidinger@biotec.tu-dresden.de; FAX 0049-351-46340348.

Article is online at http://www.genesdev.org/cgi/doi/10.1101/gad.1660508.
}

cells to dedifferentiate into progenitor cells and the activation of resident stem cells (Tanaka 2003; Slack 2006). While the cellular processes underlying blastema formation in fish are less well understood, it is possible that dedifferentiation of mesenchymal cells plays a role as well, since these cells are known to disorganize and migrate toward the wound following amputation, and no evidence has been found for the existence of long-term label-retaining stem cells in the uninjured adult fin that could be the source of blastemal cells (Nechiporuk and Keating 2002). Thus, one major goal of regeneration research is to identify the molecular mechanisms that regulate plasticity of adult tissues by converting differentiated or quiescent cells into actively proliferating tissue that undergoes pattern formation and redifferentiation.

\section{Multiple molecular signaling cascades are implemented during regeneration}

Recently, several signaling pathways have been found to regulate blastema formation in fish and amphibia (Stoick-Cooper et al. 2007a). Activin/TGF- $\beta$ signaling is required very early during zebrafish fin and amphibian limb regeneration for mesenchymal cell migration and subsequent blastema formation (Jazwinska et al. 2007; Lévesque et al. 2007). Likewise, Wnt/ $\beta$-catenin signaling is up-regulated early during fin regeneration and, in its absence, no blastema forms (Stoick-Cooper et al. 2007b), a function that is also conserved in Xenopus and salamander limb regeneration (Kawakami et al. 2006; Yokoyama et al. 2007). In addition, FGF signaling plays important roles during specification of blastemal cells. Inhibition of FGF-receptor 1 signaling by pharmacological compounds blocks blastema formation in zebrafish tail fins (Poss et al. 2000) and inhibits regeneration of Xenopus limbs (D'Jamoos et al. 1998). Most importantly, FGF signaling was confirmed to be essential for regeneration by an unbiased forward genetic screen for mutant zebrafish that fail to regenerate their tail fins. One such mutation was found to encode the FGF ligand fgf2Oa, which is up-regulated early during regeneration and is specifically required for correct wound healing and subsequent blastema formation (Whitehead et al. 2005). Activin, Wnt, and FGF signaling are also required later during regeneration for proliferation of blastemal cells (Poss et al. 2000; Jazwinska et al. 2007; Stoick-Cooper et al. 
2007b). Similarly, BMP signaling has been shown to be essential for blastema proliferation in Xenopus limb and tail regeneration (Beck et al. 2006). Despite the identification of extracellular signals that regulate progenitor cell formation and function, nothing is known about the downstream effectors that mediate the action of these pathways. Now, the study by Yin et al. (2008) shows that FGF signaling promotes blastema proliferation by inhibiting a microRNA that keeps the brakes on cell proliferation.

\section{MicroRNAs (miRNAs) in development, cell differentiation, and tissue function}

miRNAs are short, noncoding RNAs that act as negative regulators of gene expression (Bartel 2004; Bushati and Cohen 2007). In animals, most miRNAs bind to imperfectly complementary target sites in the $3^{\prime}$ untranslated regions (UTRs) of messenger RNAs and inhibit translation of the target RNA. Secondarily, this can result in destabilization of the RNA due to deadenylation. Perfectly matching miRNAs can also cause active degradation of target RNAs, but very few examples of endogenous targets of such regulation have been described in animals. Hundreds of miRNAs have been identified, with most of them being highly conserved throughout animal evolution. Based on computational approaches that screen for miRNA-binding sites in 3'UTR sequences, the emerging view is that most miRNAs have hundreds of target RNAs, and that up to $30 \%$ or more of all animal genes might be targets of miRNA regulation.

Computational and gene expression studies have suggested that genes involved in basic cellular processes have evolved to evade miRNA regulation, while genes involved in developmental processes are enriched in miRNA sites (Farh et al. 2005; Stark et al. 2005; Sood et al. 2006). The global function of miRNA can be directly tested in animals that fail to produce miRNA due to mutations in Dicer, which is essential for miRNA biosynthesis. Surprisingly, although zebrafish embryos lacking both maternally provided and zygotically produced Dicer display abnormal morphogenesis during brain, heart, and somite development, they do not show defects in early patterning or cell differentiation (Giraldez et al. 2005). In contrast to these relatively mild phenotypes, mouse embryos lacking maternally produced Dicer do not develop beyond the first cell division (Tang et al. 2007). However, tissue-specific deletion of Dicer in mice again indicates a role for miRNAs in morphogenesis and cell survival rather than in differentiation and patterning of the targeted tissues (Bushati and Cohen 2007). Based on such evidence and on the low number of miRNA mutants identified in forward genetic screens in Caenorhabditis elegans and Drosophila, it has been suggested that the functions of many miRNAs are rather subtle (Bushati and Cohen 2007).

Intuitively, it makes sense to assume that dramatic cell fate decisions and functional switches are regulated by changes in gene transcription and that an additional post-transcriptional layer of regulation by miRNAs pri- marily serves to fine-tune and stabilize expression profiles. Indeed, $m i R-430$ is required to balance expression of the Nodal agonist squint and its antagonist lefty during early zebrafish development (Choi et al. 2007). On the other hand, functional analysis of individual miRNAs has also identified some whose loss causes dramatic phenotypes, since they are involved in developmental decision making. For example, the first miRNAs identified, lin-4 and lin-7 in C. elegans, are required to progress from the first to the second larval stage (Lee et al. 1993; Wightman et al. 1993; Reinhart et al. 2000). miR-1 and miR-133 are known to play important roles during muscle differentiation and proliferation. $m i R-1$ is required for myoblast differentiation, likely because it targets histone deacetylase 4, a repressor of muscle differentiation, while $m i R-133$ promotes myoblast proliferation by repressing serum response factor (Chen et al. 2006). Knockout of one of the two miR-1 genes in mice results in defects in heart morphogenesis and cardiac cell cycle, thus confirming a role for miR-1 in muscle development in vivo (Zhao et al. 2007).

It is conceivable that the limited number of miRNAs whose function has been studied so far is biased toward those that cause dramatic, and thus easily recognizable, effects. Clearly, more studies are required to determine whether these miRNAs are an exception or whether animals use miRNAs more commonly, not just for conferring robustness to cellular decisions, but also for making choices.

\section{A role of $\mathrm{miR}-133$ in regeneration}

Here, the study by Yin et al. (2008) is the first to describe a function for miRNAs in appendage regeneration, focusing particularly on the role of $m i R-133$ in progenitor cell proliferation downstream from FGF signaling. In this case, Yin et al. (2008) study a miRNA that appears to help maintain the quiescent state of adult fin tissue and is down-regulated upon induction of regeneration to allow progenitor cell proliferation. To date, $m i R-133$ function has primarily been studied in cardiac and skeletal cells as well as in dopaminergic neurons (Chen et al. 2006; Care et al. 2007; Kim et al. 2007), while the main portion of the zebrafish fin contains no muscle nor dopaminergic neurons. Therefore, this study also represents a new departure for $m i R-133$ to participate in the transition from an adult to a regenerative tissue that primarily harbors dermal and skeletal components.

Initially, Yin et al. (2008) obtain an overview of miRNA regulation during regeneration using microarrays and Northern blotting, finding that dozens of miRNAs are induced during regeneration, and a smaller number of miRNAs are down-regulated. Expression of miRNAs has been reported before in regenerating newt lens and inner ear (Tsonis et al. 2007), where the evidence of cellular dedifferentiation and transdifferentiation is stronger than for the fish fin. In the newt lens, differentiated pigmented epithelial cells from the dorsal iris give rise to lens fibers (Tsonis and Del Rio-Tsonis 2004). The Tsonis study (Tsonis and Del Rio-Tsonis 2004) found little over- 
lap between the miRNAs that are up- or down-regulated in regenerating lens and inner-ear hair cells, except for members of the let-7 family of miRNAs, which were down-regulated during the dedifferentiation phase of regeneration in both tissues. It would be interesting to see whether these miRNAs are down-regulated in zebrafish tail fin regeneration as well. In the current study, Yin et al. (2008) do not describe the miRNA regulation data in detail, but instead focus on a subset of miRNAs that is altered after inhibiting FGF signaling during regeneration. In particular, Yin et al. (2008) target their functional studies to miR-133, which is present at high levels in uninjured fins, but strongly down-regulated in the blastema-forming phase of fin regeneration and kept at low levels throughout further regeneration.

Recently, a number of tools to intervene with miRNA function have been developed. Here, Yin et al. (2008) use these tools in combination with electroporation into the fin to up- and down-regulate miR-133 during regeneration. Overexpression of miR-133 duplexes during the outgrowth phase of fin regeneration, after the blastema has formed, results in reduced fin growth. This indicates that $m i R-133$ acts as an inhibitor of regeneration. Indeed, Yin et al. (2008) find that further reducing the already low levels of miRNA-133 during regeneration by using an antisense morpholino oligonucleotide causes slightly faster regenerate growth. Together, these data indicate that down-regulation of $m i R-133$ after amputation is required for efficient regeneration to occur. What then causes down-regulation of miR-133 expression? Yin et al. (2008) find that inhibition of FGF signaling by heatshock-inducible overexpression of a dominant-negative FGF receptor during regenerative outgrowth up-regulates miR-133 expression within a few hours. As described above, FGF signaling is required for fin regeneration; thus, if miR-133 is an important negative target of FGF signaling during regeneration, knockdown of miR-133 might rescue the defects caused by FGF pathway inhibition. Indeed, Yin et al. (2008) find that electroporation of the miR-133 morpholino allows FGF-inhibited fins to grow significantly faster than control morpholino electroporation does. It is interesting that in previous work Poss and colleagues (Lee et al. 2005) implicated FGF signaling in modulating blastema growth rate along the proximal-distal axis of the zebrafish fin. When fins are cut close to the base they grow at an accelerated rate compared with fins cut close to the tip. This difference seems to correlate with the expression level of FGF targets. It would now be important to know whether miR133 is also differentially down-regulated in a positiondependent manner during regeneration in this in vivo example of growth control.

How does miRNA-133 inhibit regenerative outgrowth? Yin et al. (2008) find that knockdown of miR133 partially rescues blastemal cell proliferation after FGF pathway inhibition. It also rescues expression of several marker genes that are down-regulated by FGF inhibition, including the homeobox transcription factor $m s x b$, which is specifically expressed in blastema cells and implicated in repression of progenitor cell differen- tiation (Poss et al. 2003). Thus, miR-133 at least partially mediates the effects of FGF signaling on proliferation of blastema cells and maintenance of their identity. The effects of $m i R-133$ on expression of $m s x b$ and the other tested markers appear to be indirect, since the $3^{\prime}$ UTRs of these genes lack predicted miR-133-binding sites.

\section{Mps1 kinase is an in vivo target of miR-133}

Finding miRNA targets is an essential and potentially difficult dimension of miRNA studies. In this case, previous mutational analysis of zebrafish regeneration comes together beautifully with the new findings. Using bioinformatics, Yin et al. (2008) identify several dozen potential direct $m i R-133$ target genes, including growth factors from the BMP and FGF family. The latter finding might indicate that miR-133 is part of a positive feedback loop, since miR-133 expression is down-regulated by FGF signaling. The potential target genes also include several cell cycle regulators, among which mps1 received the attention of Yin et al. (2008), as they had previously described its role in blastema proliferation (Poss et al. 2002a). mps1 is a kinase with a highly conserved function in the mitotic checkpoint, also called spindle checkpoint, which assures genetic stability by preventing sister chromatid separation before all chromosomes are properly aligned (Fisk et al. 2004). Remarkably, Poss et al. (2002a) discovered mps1 as essential for fin regeneration in the same genetic screen that identified fgf2Oa. During zebrafish tail fin regeneration, mps1 expression is up-regulated in a subpopulation of blastema cells, which proliferate rapidly during the outgrowth phase of regeneration (Poss et al. 2002a). Using the temperaturesensitive mutation in mps1 identified in their genetic screen, Poss et al. (2002a) showed that the kinase is required for proliferation of blastema cells and regenerative outgrowth of the fin.

Having found that mps1 RNA contains one potential target site for miR-133, Yin et al. (2008) test whether miR-133 can regulate mps1 using sensor assays in zebrafish embryos. GFP expression from a RNA construct containing the mps1 3'UTR can indeed be suppressed by coinjection of miR-133 duplexes, and the predicted miR133-binding site is required for this inhibition. Sensor RNA abundance did not change in these experiments, indicating that miR-133 regulates translation, but not stability of mps1 RNA. Interestingly, however, in regenerating fins, mps1 RNA levels are reduced after inhibition of FGF signaling, and knockdown of miR-133 can rescue this effect. Whether this discrepancy between the effects on the artificial sensor RNA in embryos and on the endogenous gene in fins reflects tissue-specific differences in miR-133 function or whether it is due to the different assays used, remains unresolved. Due to the difficulties in achieving consistent and nonmosaic delivery of RNA or DNA molecules by currently available electroporation protocols, Yin et al. (2008) did not perform sensor assays in the regenerating fin. Thus, conclusive evidence that miR-133 regulates mps 1 expression via translational inhibition and/or RNA destabilization in 
the regenerating fin in vivo has to await the establishment of transgenic fish containing sensor constructs or the development of antibodies that recognize zebrafish Mps1 protein.

\section{What is the significance and specificity of miR-133 in regenerative outgrowth?}

The work by Yin et al. (2008) raises numerous interesting questions concerning the molecular circuitry that controls regeneration. First, is miR-133 a direct target of FGF signaling, or are other signaling pathways involved in the FGF-dependent promotion of blastema proliferation through miR-133 and mps1? As mentioned previously, a number of signaling pathways are required for blastema growth, including Wnt and BMP. Yin et al. (2008) do not address whether miR-133 transcription is directly repressed by FGF signaling during regenerative outgrowth. In fact, it seems likely that another pathway could be involved: Other known targets of FGF signaling-namely, mkp3, sef, and spry4-are differently localized in the blastema compared with mps1 (Lee et al. 2005). These FGF target genes localize to the basal epidermis and the distal blastema, whereas mps1 localizes to the proximal blastema, which is the zone of proliferating cells (Poss et al. 2002a). Although Yin et al. (2008) do not describe the expression pattern of $m i R-133$, it seems fair to assume that it will be present in mps1expressing cells upon inhibition of FGF signaling. Therefore, FGF signaling may have an indirect role in repressing $m i R-133$ expression. There is some evidence that Wnt/ $\beta$-catenin signaling acts upstream of FGF signaling during fin regeneration (Stoick-Cooper et al. 2007b); thus, it would be interesting to test whether miR-133 also mediates the effects of Wnt signaling on regenerative outgrowth.

Is FGF regulation of mps1 via miR-133 specific for the regenerating fin or does this interaction also occur in other tissues and systems? Fin and limb regeneration can be viewed as a redevelopment of the structure, and it is known that some molecular regulatory logic and gene activities are shared between these two processes. FGF signaling, for example, is essential for both limb and fin development. It would thus be particularly interesting to test whether miR-133 functions downstream from FGF signaling during appendage development and whether it targets mps1 in this process. So far, expression of $m i R$ 133 during zebrafish embryogenesis has been detected in the musculature of the body, head, and fins (Wienholds et al. 2005), but detailed mapping of the expression pattern in the developing fin is required to determine whether it is down-regulated in proliferative zones as predicted if it has a similar role as during adult fin regeneration. Yin et al. (2008) report that the adult zebrafish heart expresses high levels of miR-133. Intriguingly, mps1 is also required for heart regeneration (Poss et al. 2002b), raising the possibility that miR-133 downregulation and subsequent activation of mps1 expression are also prerequisites for cardiomyocyte expansion during zebrafish heart regeneration. In mammals, the heart does not regenerate, since injury does not activate cardiomyocyte proliferation but rather results in cardiac hypertrophy (Murry et al. 2006). In mouse, miR-133 was found to inhibit cardiac hypertrophy, and remarkably, knockdown of miR-133 by infusion of an antisense oligo was sufficient to cause sustained hypertrophy (Care et al. 2007). It is therefore possible that $m i R-133$ is required to suppress cardiomyocyte activation in both the zebrafish and mammalian heart, but perhaps it regulates different targets, which might help explain why zebrafish hearts can regenerate, while mammalian hearts cannot. The context-specific functions of $m i R-133$ are likely to be an important consideration for future studies since, for example, miR-133 promotes proliferation of mouse skeletal myoblasts in culture (Chen et al. 2006), whereas Yin et al. (2008) show that it inhibits cell proliferation in the fish fin.

\section{Are miRNAs a means of retaining growth potential in adult tissue?}

Why does regulation of regeneration involve miRNAs? A rather straightforward explanation would be that regenerative processes require rapid and rather large-scale changes in gene expression patterns. Because miRNAs typically have many targets, they could preferentially be used in such situations. It is conceivable that during dedifferentiation, cells up-regulate miRNA expression to rapidly repress genes associated with the differentiated phenotype. In support of this idea, Yin et al. (2008) find that a considerably higher number of miRNAs are upregulated during tail fin regeneration than down-regulated. It would be interesting to analyze whether their targets include differentiation markers. On the other hand, developmental biology provides examples in which transcriptional regulation of a single master regulatory gene is sufficient to induce formation of a whole organ, involving transcriptional changes in hundreds of genes, such as induction of ectopic eyes by pax6 (Halder et al. 1995). Thus, whether or not the necessity to regulate many genes is an explanation for the involvement of miRNAs is somewhat doubtful. On the other hand, master transcriptional regulators that induce regeneration have not been identified, and perhaps reactivation of structure development from differentiated tissue, rather than undifferentiated embryonic cells, is less likely to involve such regulators.

Are miRNAs merely required to fine-tune gene expression programs during regeneration, or are they major instructive factors? The latter might be true for miR-133. Yin et al. (2008) show that loss of miR-133 is sufficient to rescue the cell proliferation defects caused by inhibiting FGF signaling to a significant extent. This experimental result might actually underestimate the importance of miR-133 as an FGF effector because the miR-133 knockdown was most likely a partial phenotype due to the generally incomplete gene knockdown by morpholino electroporation into the fin. Clearly, more studies are needed to test whether loss of other miRNAs produces significant phenotypes and whether miRNAs play roles 
in cell dedifferentiation and cell fate decisions during regeneration.

An interesting speculation is that regulation of gene expression by post-transcriptional mechanisms might aid in maintaining the plasticity of regenerating tissues. Many regenerative processes are known or assumed to involve dedifferentiation of cells into progenitors that can give rise to other differentiated cell types. Thus, in order to maintain such plasticity, tissues that are competent to regenerate might have to avoid mechanisms of transcriptional regulation that are difficult to erase, such as chromatin modifications, and instead rely on posttranscriptional mechanisms of gene regulation. Maybe fins need to ensure that genes required for regeneration, such as mps1, are accessible for fast up-regulation in the event of wounding. One way to assure this might be to shield these genes from silencing chromatin modifications, although this increases the risk of detrimental effects from leaky expression in noninjured tissue. Hence the need to repress expression of such genes by miRNAs in the noninjured tissue. An interesting experiment along these lines would be to assess the consequences of miR-133 knockdown in nonamputated fins. Considering that mps1 expression cannot be detected at the RNA level in nonamputated fins (Poss et al. 2002a), it is worth determining whether this regulation is due to repression by $\operatorname{miR}-133$.

Adult zebrafish clearly retain tissue plasticity even under nonregenerative conditions, as they display indeterminate growth-becoming larger if they are put in a bigger tank. In a separate study, Poss and colleagues (Wills et al. 2008) utilized the heart model to explore some of the cellular processes underlying tissue regulation. When zebrafish are transferred into growth conditions, cells in various heart compartments begin to proliferate, which includes the production of new cardiomyocytes. Using overexpression of the dominant-negative FGF receptor, Wills et al. (2008) showed that this signaling pathway is involved in cardiac homeostasis. It would not be surprising if the FGF/miR-133/mps1 pathway also acts in this scenario. The miRNAs may well be important for repressing proliferation, but maintaining the potential to proliferate and activate embryonic gene programs in adult tissues, or subsets of cells within mature tissues, to allow growth and regeneration. It will be fascinating to see whether the mechanisms of tissue homeostasis seen in zebrafish and other indeterminate growers are common or distinct to the mechanisms found in every vertebrate.

A specific hypothesis would be to investigate whether miRNAs represent important points of evolutionary change that determine different growth control traits and whether this knowledge can be used to confer more tissue plasticity to other vertebrate tissues.

\section{Acknowledgments}

We thank Laurel Rhode for critical reading of the manuscript. Work in G.W.'s laboratory is funded by the Collaborative Research Center SFB 655 "Cells into tissues" of the Deutsche Forschungsgemeinschaft.

\section{References}

Bartel, D.P. 2004. MicroRNAs: Genomics, biogenesis, mechanism, and function. Cell 116: 281-297.

Beck, C.W., Christen, B., Barker, D., and Slack, J.M. 2006. Temporal requirement for bone morphogenetic proteins in regeneration of the tail and limb of Xenopus tadpoles. Mech. Dev. 123: 674-688.

Bushati, N. and Cohen, S.M. 2007. microRNA functions. Annu. Rev. Cell Dev. Biol. 23: 175-205.

Care, A., Catalucci, D., Felicetti, F., Bonci, D., Addario, A., Gallo, P., Bang, M.L., Segnalini, P., Gu, Y., Dalton, N.D., et al. 2007. MicroRNA-133 controls cardiac hypertrophy. Nat. Med. 13: 613-618.

Chen, J.F., Mandel, E.M., Thomson, J.M., Wu, Q., Callis, T.E., Hammond, S.M., Conlon, F.L., and Wang, D.Z. 2006. The role of microRNA-1 and microRNA-133 in skeletal muscle proliferation and differentiation. Nat. Genet. 38: 228-233.

Choi, W.Y., Giraldez, A.J., and Schier, A.F. 2007. Target protectors reveal dampening and balancing of Nodal agonist and antagonist by miR-430. Science 318: 271-274.

D'Jamoos, C.A., McMahon, G., and Tsonis, P.A. 1998. Fibroblast growth factor receptors regulate the ability for hindlimb regeneration in Xenopus laevis. Wound Repair Regen. 6: 388-397.

Farh, K.K., Grimson, A., Jan, C., Lewis, B.P., Johnston, W.K., Lim, L.P., Burge, C.B., and Bartel, D.P. 2005. The widespread impact of mammalian microRNAs on mRNA repression and evolution. Science 310: 1817-1821.

Fisk, H.A., Mattison, C.P., and Winey, M. 2004. A field guide to the Mps1 family of protein kinases. Cell Cycle 3: 439-442.

Giraldez, A.J., Cinalli, R.M., Glasner, M.E., Enright, A.J., Thomson, J.M., Baskerville, S., Hammond, S.M., Bartel, D.P., and Schier, A.F. 2005. MicroRNAs regulate brain morphogenesis in zebrafish. Science 308: 833-838.

Halder, G., Callaerts, P., and Gehring, W.J. 1995. Induction of ectopic eyes by targeted expression of the eyeless gene in Drosophila. Science 267: 1788-1792.

Jazwinska, A., Badakov, R., and Keating, M.T. 2007. Activin- $\beta$ A signaling is required for zebrafish fin regeneration. Curr. Biol. 17: 1390-1395.

Kawakami, Y., Rodriguez Esteban, C., Raya, M., Kawakami, H., Marti, M., Dubova, I., and Izpisua Belmonte, J.C. 2006. Wnt/ $\beta$-catenin signaling regulates vertebrate $\mathrm{limb}$ regeneration. Genes \& Dev. 20: 3232-3237.

Kim, J., Inoue, K., Ishii, J., Vanti, W.B., Voronov, S.V., Murchison, E., Hannon, G., and Abeliovich, A. 2007. A microRNA feedback circuit in midbrain dopamine neurons. Science 317: 1220-1224.

Lee, R.C., Feinbaum, R.L., and Ambros, V. 1993. The C. elegans heterochronic gene lin-4 encodes small RNAs with antisense complementarity to lin-14. Cell 75: 843-854.

Lee, Y., Grill, S., Sanchez, A., Murphy-Ryan, M., and Poss, K.D. 2005. Fgf signaling instructs position-dependent growth rate during zebrafish fin regeneration. Development 132: 51735183.

Lévesque, M., Gatien, S., Finnson, K., Desmeules, S., Villiard, E., Pilote, M., Philip, A., and Roy, S. 2007. Transforming growth factor: $\beta$ signaling is essential for limb regeneration in axolotls. PloS ONE 2: e1227. doi: 10.1371/journal.pone/ 0001227.

Murry, C.E., Reinecke, H., and Pabon, L.M. 2006. Regeneration gaps: Observations on stem cells and cardiac repair. J. Am. Coll. Cardiol. 47: 1777-1785.

Nechiporuk, A. and Keating, M.T. 2002. A proliferation gradient between proximal and msxb-expressing distal blastema 
directs zebrafish fin regeneration. Development 129: 26072617.

Poss, K.D., Shen, J., Nechiporuk, A., McMahon, G., Thisse, B., Thisse, C., and Keating, M.T. 2000. Roles for Fgf signaling during zebrafish fin regeneration. Dev. Biol. 222: 347-358.

Poss, K.D., Nechiporuk, A., Hillam, A.M., Johnson, S.L., and Keating, M.T. 2002a. Mps1 defines a proximal blastemal proliferative compartment essential for zebrafish fin regeneration. Development 129: 5141-5149.

Poss, K.D., Wilson, L.G., and Keating, M.T. 2002b. Heart regeneration in zebrafish. Science 298: $2188-2190$.

Poss, K.D., Keating, M.T., and Nechiporuk, A. 2003. Tales of regeneration in zebrafish. Dev. Dyn. 226: 202-210.

Reinhart, B.J., Slack, F.J., Basson, M., Pasquinelli, A.E., Bettinger, J.C., Rougvie, A.E., Horvitz, H.R., and Ruvkun, G. 2000. The 21-nucleotide let-7 RNA regulates developmental timing in Caenorhabditis elegans. Nature 403: 901-906.

Slack, J.M. 2006. Amphibian muscle regeneration-Dedifferentiation or satellite cells? Trends Cell Biol. 16: 273-275.

Sood, P., Krek, A., Zavolan, M., Macino, G., and Rajewsky, N. 2006. Cell-type-specific signatures of microRNAs on target mRNA expression. Proc. Natl. Acad. Sci. 103: 2746-2751.

Stark, A., Brennecke, J., Bushati, N., Russell, R.B., and Cohen, S.M. 2005. Animal microRNAs confer robustness to gene expression and have a significant impact on 3'UTR evolution. Cell 123: 1133-1146.

Stoick-Cooper, C.L., Moon, R.T., and Weidinger, G. 2007a. Advances in signaling in vertebrate regeneration as a prelude to regenerative medicine. Genes \& Dev. 21: 1292-1315.

Stoick-Cooper, C.L., Weidinger, G., Riehle, K.J., Hubbert, C., Major, M.B., Fausto, N., and Moon, R.T. 2007b. Distinct Wnt signaling pathways have opposing roles in appendage regeneration. Development 134: 479-489.

Tanaka, E.M. 2003. Cell differentiation and cell fate during urodele tail and limb regeneration. Curr. Opin. Genet. Dev. 13: 497-501.

Tang, F., Kaneda, M., O'Carroll, D., Hajkova, P., Barton, S.C., Sun, Y.A., Lee, C., Tarakhovsky, A., Lao, K., and Surani, M.A. 2007. Maternal microRNAs are essential for mouse zygotic development. Genes \& Dev. 21: 644-648.

Tsonis, P.A. and Del Rio-Tsonis, K. 2004. Lens and retina regeneration: Transdifferentiation, stem cells and clinical applications. Exp. Eye Res. 78: 161-172.

Tsonis, P.A., Call, M.K., Grogg, M.W., Sartor, M.A., Taylor, R.R., Forge, A., Fyffe, R., Goldenberg, R., Cowper-Sal-lari, R., and Tomlinson, C.R. 2007. MicroRNAs and regeneration: Let- 7 members as potential regulators of dedifferentiation in lens and inner ear hair cell regeneration of the adult newt. Biochem. Biophys. Res. Commun. 362: 940-945.

Whitehead, G.G., Makino, S., Lien, C.L., and Keating, M.T. 2005. fgf20 is essential for initiating zebrafish fin regeneration. Science 310: 1957-1960.

Wienholds, E., Kloosterman, W.P., Miska, E., Alvarez-Saavedra, E., Berezikov, E., de Bruijn, E., Horvitz, H.R., Kauppinen, S., and Plasterk, R.H. 2005. MicroRNA expression in zebrafish embryonic development. Science 309: 310-311.

Wightman, B., Ha, I., and Ruvkun, G. 1993. Posttranscriptional regulation of the heterochronic gene lin-14 by lin- 4 mediates temporal pattern formation in C. elegans. Cell 75: 855-862.

Wills, A.A., Holdway, J.E., Major, R.J., and Poss, K.D. 2008. Regulated addition of new myocardial and epicardial cells fosters homeostatic cardiac growth and maintenance in adult zebrafish. Development 135: 183-192.

Yin, V.P., Thomson, J.M., Thummel, R., Hyde, D.R., Hammond, S.M., and Poss, K.D. 2008. Fgf-dependent depletion of microRNA-133 promotes appendage regeneration in ze- brafish. Genes \& Dev. (this issue). doi: 10.1101/gad.1641808.

Yokoyama, H., Ogino, H., Stoick-Cooper, C.L., Grainger, R.M., and Moon, R.T. 2007. Wnt/ $\beta$-catenin signaling has an essential role in the initiation of limb regeneration. Dev. Biol. 306: 170-178.

Zhao, Y., Ransom, J.F., Li, A., Vedantham, V., von Drehle, M., Muth, A.N., Tsuchihashi, T., McManus, M.T., Schwartz, R.J., and Srivastava, D. 2007. Dysregulation of cardiogenesis, cardiac conduction, and cell cycle in mice lacking miRNA1-2. Cell 129: 303-317. 


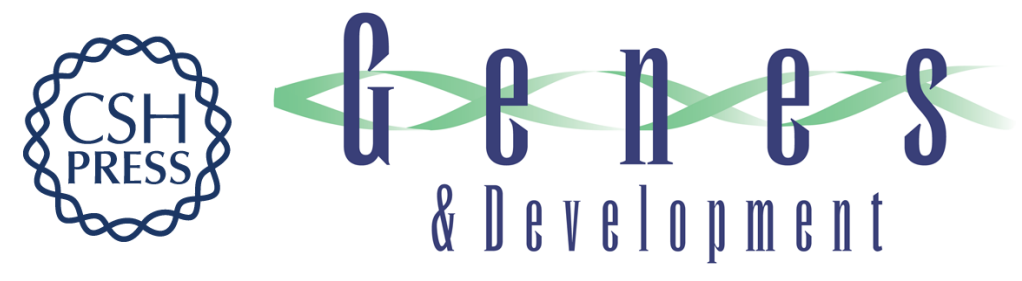

\section{Micromanaging regeneration}

Elly M. Tanaka and Gilbert Weidinger

Genes Dev. 2008, 22:

Access the most recent version at doi:10.1101/gad.1660508

Related Content Fgf-dependent depletion of microRNA-133 promotes appendage regeneration in zebrafish

Viravuth P. Yin, J. Michael Thomson, Ryan Thummel, et al.

Genes Dev. March , 2008 22: 728-733

References This article cites 40 articles, 17 of which can be accessed free at:

http://genesdev.cshlp.org/content/22/6/700.full.html\#ref-list-1

Articles cited in:

http://genesdev.cshlp.org/content/22/6/700.full.html\#related-urls

\section{License}

Email Alerting Receive free email alerts when new articles cite this article - sign up in the box at the top Service right corner of the article or click here.

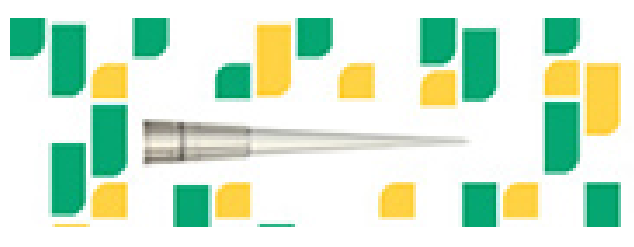

Focused on your science. 